\title{
ON REGULAR HOMEOMORPHISMS IN THE PLANE
}

\section{Ruslan Salimov}

National Academy of Sciences of Ukraine, Institute of Applied Mathematics and Mechanics 74 Roze Luxemburg str., 83114 Donetsk, Ukraine; salimov@iamm.ac.donetsk.ua

\begin{abstract}
A regular homeomorphism of the Sobolev class $W_{\text {loc }}^{1,1}$ in the plane domain $D$ is a ring $Q$-homeomorphism with $Q(z)=K_{\mu}^{T}\left(z, z_{0}\right)$ where $K_{\mu}^{T}\left(z, z_{0}\right)$ is the tangential dilatation of $f$ at $z_{0} \in D$.
\end{abstract}

\section{Introduction}

It has been established in [Sal] that a $Q$-homeomorphism in $\mathbf{R}^{n}, n \geq 2$, is in $W_{\text {loc }}^{1,1}$ and differentiable with its Jacobian $J_{f}(z) \neq 0$ a.e. whenever $Q \in L_{\text {loc }}^{1}$. These results were extended to ring $Q$-homeomorphisms in $\left[\mathrm{SS}_{1}\right]$ and $\left[\mathrm{SS}_{2}\right]$. In the present paper it is conversely stated that every homeomorphism in the plane of the class $W_{\text {loc }}^{1,1}$ with $J_{f}(z)>0$ a.e. is a ring $Q$-homeomorphism with $Q(z)=K_{f}(z)$. Moreover, we give a pointwise characterization of this property.

Let $D$ be a domain in the complex plane $\mathbf{C}$, i.e., a connected and open subset of C. In what follows, we call a homeomorphism $f: D \rightarrow \mathbf{C}$ of the class $W_{\text {loc }}^{1,1}$ regular if $J_{f}(z)>0$ a.e. Note that every regular homeomorphism satisfies a Beltrami equation.

The Beltrami equation is the equation of the form

$$
f_{\bar{z}}=\mu(z) \cdot f_{z}
$$

where $f_{\bar{z}}=\bar{\partial} f=\left(f_{x}+i f_{y}\right) / 2, f_{z}=\partial f=\left(f_{x}-i f_{y}\right) / 2, z=x+i y$, and $f_{x}$ and $f_{y}$ are partial derivatives of $f$ in $x$ and $y$, correspondingly, and $\mu: D \rightarrow \mathbf{C}$ be a measurable function with $|\mu(z)|<1$ a.e. The function $\mu$ is called the complex coefficient and

$$
K_{\mu}(z)=\frac{1+|\mu(z)|}{1-|\mu(z)|}
$$

the dilatation of the equation (1.1). The Beltrami equation (1.1) is said to be degenerate if $\operatorname{ess} \sup K_{\mu}(z)=\infty$.

Given a point $z_{0}$ in $\bar{D}$, the tangential dilatation of (1.1) with respect to $z_{0}$ is the function

$$
K_{\mu}^{T}\left(z, z_{0}\right)=\frac{\left|1-\frac{\overline{z-z_{0}}}{z-z_{0}} \mu(z)\right|^{2}}{1-|\mu(z)|^{2}},
$$

see $\left[\mathrm{RSY}_{1}\right]-\left[\mathrm{RSY}_{2}\right]$, cf. the corresponding terms and notations in $\left[\mathrm{An}_{1}\right]-\left[\mathrm{An}_{3}\right],[\mathrm{Ch}]$, [GMSV], [Le] and [RW]. If $f$ is a regular homeomorphism, then for a.e. $z \in D$

$$
K_{\mu}^{T}\left(z, z_{0}\right)=\frac{\left|f_{\theta}(z)\right|^{2}}{r^{2} J_{f}(z)}
$$

doi:10.5186/aasfm.2010.3517

2000 Mathematics Subject Classification: Primary 30C65; Secondary 30C75.

Key words: Ring $Q$-homeomorphisms, regular homeomorphisms, modulus, absolute continuity on lines, differentiability, Sobolev's classes. 
where $z=z_{0}+r e^{i \theta}$, see, e.g., $\left[\operatorname{RSY}_{1},(2.4)\right]$.

Recall that a function $f: D \rightarrow \mathbf{C}$ is absolutely continuous on lines, abbr. $f \in$ ACL, if, for every closed rectangle $R$ in $D$ whose sides are parallel to the coordinate axes, $f \mid R$ is absolutely continuous on almost all line segments in $R$ which are parallel to the sides of $R$. In particular, $f$ is ACL (possibly modified on a set of Lebesgue measure zero) if it belongs to the Sobolev class $W_{\text {loc }}^{1,1}$ of locally integrable functions with locally integrable first generalized derivatives and, conversely, if $f \in$ ACL has locally integrable first partial derivatives, then $f \in W_{\text {loc }}^{1,1}$, see, e.g., [MP, 1.2.4]. Note that, if $f \in$ ACL, then $f$ has partial derivatives $f_{x}$ and $f_{y}$ a.e. and, for a sensepreserving ACL homeomorphism $f: D \rightarrow \mathbf{C}$, the Jacobian $J_{f}(z)=\left|f_{z}\right|^{2}-\left|f_{\bar{z}}\right|^{2}$ is nonnegative a.e. In this case, the complex dilatation $\mu_{f}$ of $f$ is the ratio $\mu(z)=f_{\bar{z}} / f_{z}$, if $f_{z} \neq 0$ and $\mu(z)=0$ otherwise, and the dilatation $K_{f}$ of $f$ is $K_{\mu}(z)$, see (1.2).

\section{On ring $Q$-homeomorphisms}

Recall that, given a family of paths $\Gamma$ in $\overline{\mathbf{C}}$, a Borel function $\rho: \overline{\mathbf{C}} \rightarrow[0, \infty]$ is called admissible for $\Gamma$, abbr. $\rho \in \operatorname{adm} \Gamma$, if

$$
\int_{\gamma} \rho(z)|d z| \geq 1
$$

for each $\gamma \in \Gamma$. The modulus of $\Gamma$ is defined by

$$
M(\Gamma)=\inf _{\rho \in \operatorname{adm} \Gamma} \int_{\mathbf{C}} \rho^{2}(z) d x d y .
$$

Given a domain $D$ and two sets $E$ and $F$ in $\overline{\mathbf{C}}, \Delta(E, F, D)$ denotes the family of all paths $\gamma:[a, b] \rightarrow \overline{\mathbf{C}}$ which join $E$ and $F$ in $D$, i.e., $\gamma(a) \in E, \gamma(b) \in F$ and $\gamma(t) \in D$ for $a<t<b$. We set $\Delta(E, F)=\Delta(E, F, \overline{\mathbf{C}})$ if $D=\overline{\mathbf{C}}$. Recall that a ring domain, or shortly a ring in $\overline{\mathbf{C}}$, is a domain $R$ whose complement $\overline{\mathbf{C}} \backslash R$ consists of two components.

Motivated by the ring definition of quasiconformality in [Ge], the following notion was introduced in $\left[\mathrm{RSY}_{1}\right]-\left[\mathrm{RSY}_{2}\right]$. Let $D$ be a domain in $\mathbf{C}, z_{0} \in D$, and $Q: D \rightarrow$ $[0, \infty]$ a measurable function. A homeomorphism $f: D \rightarrow \mathbf{C}$ is called a ring $Q$ homeomorphism at the point $z_{0}$ if

$$
M\left(\Delta\left(f C_{1}, f C_{2}, f D\right)\right) \leq \int_{A} Q(z) \cdot \eta^{2}\left(\left|z-z_{0}\right|\right) d x d y
$$

for every ring

$$
A=A\left(z_{0}, r_{1}, r_{2}\right)=\left\{z \in \mathbf{C}: r_{1}<\left|z-z_{0}\right|<r_{2}\right\}, 0<r_{1}<r_{2}<\operatorname{dist}\left(z_{0}, \partial D\right),
$$

and every measurable function $\eta:\left(r_{1}, r_{2}\right) \rightarrow[0, \infty]$ such that

$$
\int_{r_{1}}^{r_{2}} \eta(r) d r=1
$$

and where $C_{1}=\left\{z \in \mathbf{C}:\left|z-z_{0}\right|=r_{1}\right\}$ and $C_{2}=\left\{z \in \mathbf{C}:\left|z-z_{0}\right|=r_{2}\right\}$.

Recall a criterion of ring $Q$-homeomorphisms obtained in [RS, Theorem 2.1], see also [MRSY, Theorem 7.2]. Below we use the standard conventions $a / \infty=0$ for $a \neq \infty$ and $a / 0=\infty$ if $a>0$ and $0 \cdot \infty=0$, see, e.g., [Sa, p. 6]. 
Lemma 2.1. Let $D$ be a domain in $\mathbf{C}$ and $Q: D \rightarrow[0, \infty]$ a measurable function. A homeomorphism $f: D \rightarrow \mathbf{C}$ is a ring $Q$-homeomorphism at a point $z_{0} \in D$ if and only if for every $0<r_{1}<r_{2}<d_{0}=\operatorname{dist}\left(z_{0}, \partial D\right)$,

$$
M\left(\Delta\left(f C_{1}, f C_{2}, f D\right)\right) \leq \frac{2 \pi}{I},
$$

where $C_{1}=\left\{z \in \mathbf{C}:\left|z-z_{0}\right|=r_{1}\right\}, C_{2}=\left\{z \in \mathbf{C}:\left|z-z_{0}\right|=r_{2}\right\}$ and

$$
I=I\left(r_{1}, r_{2}\right)=\int_{r_{1}}^{r_{2}} \frac{d r}{r q_{z_{0}}(r)}
$$

where $q_{z_{0}}(r)$ is the mean value of $Q(z)$ over the circle $\left|z-z_{0}\right|=r$.

Note that the infimum from the right hand side in (2.3) holds for the function

$$
\eta_{0}(r)=\frac{1}{\operatorname{Irq} q_{z_{0}}(r)}
$$

\section{The main results}

Theorem 3.1. Let $f: D \rightarrow \mathbf{C}$ be a regular homeomorphism. Then $f$ is a ring $Q$-homeomorphisms at a point $z_{0} \in D$ with $Q(z)=K_{\mu}^{T}\left(z, z_{0}\right), \mu=\mu_{f}$.

Proof. Without loss of generality, we may assume that $z_{0}=0 \in D$. Consider the ring $R=\left\{z \in \mathbf{C}: r_{1}<|z|<r_{2}\right\}$. Then there is a conformal map $h$ mapping the ring $f R$ onto a ring $R^{*}=\{w: 1<|w|<L\}$.

Let $\Gamma^{*}$ be the family of paths joining boundary components $|w|=1$ and $|w|=L$ of the ring $R^{*}$. Then, in view of conformal invariantnce of modulus, $M\left(\Gamma^{*}\right)=M(\Gamma)$, where $\Gamma$ is the family of all path joining the boundary components of the ring $f R$. Thus,

$$
M(\Gamma)=\frac{4 \pi^{2}}{\int_{R^{*}} \frac{d u d v}{|w|^{2}}} .
$$

Denote by $C_{r}, r_{1}<r<r_{2}$, circles $\{z:|z|=r\}$. For $g=h \circ f$, we have that $g \in W_{\text {loc }}^{1,1}(R)$, and hence $g$ is a.e. differentiable and absolutely continuous on $C_{r}$ for a.e. $r \in\left(r_{1}, r_{2}\right)$. The latter follows from the invariance of the class $W_{\text {loc }}^{1,1}$ under locally quasi-isometric transformations of coordinates, see, e.g., [Ma, 1.1.7]. Note that

$$
\int_{r_{1}}^{r_{2}} \int_{0}^{2 \pi} \frac{J_{g}\left(r e^{i \theta}\right)}{\left|g\left(r e^{i \theta}\right)\right|^{2}} r d r d \theta \leq \int_{R^{*}} \frac{d u d v}{|w|^{2}}=\frac{(2 \pi)^{2}}{M(\Gamma)},
$$

where $w=u+i v, J_{g}$ is the Jacobian of $g$, see, e.g., [LV, Lemma III.3.3].

Now, we have

$$
2 \pi \leq \int_{C_{r}}|d \arg g| \leq \int_{C_{r}} \frac{|d g(z)|}{|g(z)|}=\int_{0}^{2 \pi} \frac{\left|g_{\theta}\left(r e^{i \theta}\right)\right|}{\left|g\left(r e^{i \theta}\right)\right|} d \theta
$$


for a.e. $r \in\left(r_{1}, r_{2}\right)$ and applying the Schwarz inequality, see, e.g., [BB, Theorem I.4], we obtain that

$$
(2 \pi)^{2} \leq\left(\int_{0}^{2 \pi} \frac{\left|g_{\theta}\left(r e^{i \theta}\right)\right|}{\left|g\left(r e^{i \theta}\right)\right|} d \theta\right)^{2} \leq \int_{0}^{2 \pi} \frac{\left|g_{\theta}\left(r e^{i \theta}\right)\right|^{2}}{J\left(r e^{i \theta}\right)} d \theta \int_{0}^{2 \pi} \frac{J\left(r e^{i \theta}\right)}{\left|g\left(r e^{i \theta}\right)\right|^{2}} d \theta
$$

i.e.,

$$
\frac{2 \pi}{r \frac{1}{2 \pi} \int_{0}^{2 \pi} \frac{\left|g \theta\left(r e^{i \theta}\right)\right|^{2}}{r^{2} J\left(r e^{i \theta}\right)} d \theta} \leq r \int_{0}^{2 \pi} \frac{J\left(r e^{i \theta}\right)}{\left|g\left(r e^{i \theta}\right)\right|^{2}} d \theta .
$$

Setting, see (1.4),

$$
k(r)=\frac{1}{2 \pi} \int_{0}^{2 \pi} \frac{\left|g_{\theta}\left(r e^{i \theta}\right)\right|^{2}}{r^{2} J\left(r e^{i \theta}\right)} d \theta=\frac{1}{2 \pi r} \int_{C_{r}} K_{\mu}^{T}\left(z, z_{0}\right)|d z|
$$

and, integrating the both sides of the inequality (3.2) over $r \in\left(r_{1}, r_{2}\right)$, we see that

$$
2 \pi \int_{r_{1}}^{r_{2}} \frac{d r}{r k(r)} \leq \int_{r_{1}}^{r_{2}} r d r \int_{0}^{2 \pi} \frac{J\left(r e^{i \theta}\right)}{\left|g\left(r e^{i \theta}\right)\right|^{2}} d \theta
$$

Combining the last inequality and (3.1), we have by the Fubini theorem that

$$
\int_{r_{1}}^{r_{2}} \frac{d r}{r k(r)} \leq \frac{2 \pi}{M(\Gamma)}
$$

Thus,

$$
M(\Gamma) \leq \frac{2 \pi}{\int_{r_{1}}^{r_{2}} \frac{d r}{\left.r k_{(} r\right)}}
$$

Finally, applying Lemma 2.1, we obtain the conclusion of the theorem.

Corollory 3.1. Every regular homeomorphism $f: D \rightarrow \mathbf{C}$ is a ring Q-homeomorphism with $Q(z)=K_{\mu}(z), \mu=\mu_{f}$, at each point $z_{0} \in D$.

Thus, the theory of ring $Q$-homeomorphisms can be applied to regular homeomorphisms of the Sobolev class $W_{\text {loc }}^{1,1}$ in the plane, see, e.g., [MRSY, Chapter 7].

\section{References}

[An 1 Andreian Cazacu, C.: Sur les transformations pseudo-analytiques. - Rev. Math. Pures Appl. 2, 1957, 383-397.

[An 2 Andreian CAzACU, C.: Sur les ralations entre les functions caracteristiques de la pseudoanalyticite. - In: Lucrarile celui de al IV-lea Congres al Matematicienilor Romani, Bucuresti, 1956.

[An 3 Andreian CAzACU, C.: On the length-area dilatation. - Complex Var. Theory Appl. 50:7-11, 2005, 765-776.

[BB] Beckenbach, E. F., and R. Bellman: Inequalities. - Springer, New York, 1965.

[Ch] Chen, Z. G.: $\mu(z)$-homeomorphisms of the plane. - Michigan Math. J. 51:3, 2003, 547-556. 
[GMSV] Gutlyanskiı̆, V., O. Martio, T. Sugava, and M. Vuorinen: On the degenerate Beltrami equation. - Trans. Amer. Math. Soc. 357:3, 2005, 875-900.

[Ge] Gehring, F. W.: Rings and quasiconformal mappings in space. - Trans. Amer. Math. Soc. 103, 1962, 353-393.

[Le] Lehto, O.: Homeomorphisms with a prescribed dilatation. - Lecture Notes in Math. 118, $1968,58-73$.

[LV] Lehto, O., and K. I. Virtanen: Quasiconformal mappings in the plane. - SpringerVerlag, New York-Heidelberg, 1973.

[MrSY] Martio, O., V. Ryazanov, U. Srebro, and E. Yakubov: Moduli in modern mapping theory. - Springer Monogr. Math., Springer, New York, 2009.

[Ma] MaZ'Ya, V.: Sobolev classes. - Springer, Berlin-New York, 1985.

[MP] Maz'ya, V. G., and S. V. Poborchi: Differentiable functions on bad domains. - Singapure-New Jersey-London-Hong Kong, World Scientific, 1997.

[RS] Ryazanov, V.I., and E. A. Sevostyanov: Equicontinuous classes of ring $Q$-homeomorphisms. - Sibirsk. Mat. Zh. 48:6, 2007, 1361-1376 (in Russian); English transl. in Siberian Math. J. 48:6, 2007, 1093-1105.

[RSY $\left.{ }_{1}\right]$ Ryazanov, V., U. Srebro, and E. Yakubov: On ring solutions of Beltrami equations. - J. Anal. Math. 96, 2005, 117-150.

$\left[\mathrm{RSY}_{2}\right]$ Ryazanov, V., U. Srebro, and E. Yakubov: Degenerate Beltrami equation and radial Q-homeomorphisms. - Reports in Math. 369, Univ. of Helsinki, 2003, 1-34.

[RW] REICH, E., and H. WALCZAK: On the behavior of quasiconformal mappings at a point. Trans. Amer. Math. Soc. 117, 1965, 338-351.

[Sa] SaKs, S.: Theory of the integral. - Dover Publ. Inc., New York, 1964.

[Sal] Salimov, R.: ACL and differentiability of $Q$-homeomorphisms. - Ann. Acad. Sci. Fenn. Math. 33, 2008, 295-301.

$\left[\mathrm{SS}_{1}\right]$ Salimov, R., and E. Sevostyanov: ACL and differentiability of ring $Q$-homeomorphisms. - Proceeding of IAMM, NAS of Ukraine 16, 2008, 171-178.

[SS $\left.\mathrm{SS}_{2}\right]$ Salimov, R., and E. Sevostyanov: ACL and differentiability of the open discrete ring mappings. - Complex Var. Elliptic Equ. 55:1-3, 2010, 49-59. 